\title{
BMJ Open Health and Prevention Enhancement (H-PEACE): a retrospective, population- based cohort study conducted at the Seoul National University Hospital Gangnam Center, Korea
}

Changhyun Lee, ${ }^{1}$ Eun Kyung Choe, ${ }^{1}$ Ji Min Choi, ${ }^{1}$ Yunji Hwang, ${ }^{2,3,4}$ Young Lee, ${ }^{1}$ Boram Park, ${ }^{1}$ Su Jin Chung, ${ }^{1}$ Min-Sun Kwak, ${ }^{1}$ Jong-Eun Lee, ${ }^{5}$ Joo Sung Kim, ${ }^{1}$ Sue Kyung Park, ${ }^{2,3,4}$ Sang-Heon Cho ${ }^{1}$

To cite: Lee C, Choe EK, Choi JM, et al. Health and Prevention Enhancement (H-PEACE): a retrospective, population-based cohort study conducted at the Seoul National University Hospital Gangnam Center, Korea. BMJ Open 2018;8:e019327. doi:10.1136/ bmjopen-2017-019327

- Prepublication history for this paper is available online. To view these files, please visit the journal online (http://dx.doi. org/10.1136/bmjopen-2017019327).

SKP and S-HC contributed equally.

$\mathrm{CL}$ and EKC contributed equally.

Received 25 August 2017 Revised 12 February 2018 Accepted 19 March 2018
Check for updates

For numbered affiliations see end of article.

Correspondence to Dr Sang-Heon Cho; shcho@snu.ac.kr

\section{ABSTRACT}

Purpose The Health and Prevention Enhancement (H-PEACE) study was designed to investigate the association of diagnostic imaging results, biomarkers and the predisease stage of non-communicable diseases (NCDs), such as malignancies and metabolic diseases, in an average-risk population in Korea.

Participants This study enrolled a large-scale retrospective cohort at the Healthcare System Gangnam Center, Seoul National University Hospital, from October 2003 to December 2014.

Findings to date The baseline and follow-up information collected in the predisease stage of NCDs allows for evaluation of an individual's potential NCD risk, which is necessary for establishing personalised prevention strategies. A total of 91336 health examinees were included in the cohort, and we repeatedly measured and collected information for $50.9 \%$ ( $n=46484$ ) of the cohort members. All participants completed structured questionnaires (lifestyle, medical history, mini-dietary assessment index, sex-specific variables and psychiatric assessment), doctors' physical examinations, laboratory blood and urine tests and digital chest X-ray imaging. For participants with available data, we also obtained information on specific diagnostic variables using advanced diagnostic tests, including coronary CT for coronary calcium scores, colonoscopy and brain MRI. Furthermore, 17455 of the participants who provided informed consent and donated blood samples were enrolled into the Gene-environmental interaction and phenotype study, a subcohort of the H-PEACE, from October 2013, and we analysed genome-wide singlenucleotide polymorphism array data for 6579 of these blood samples.

Future plans The data obtained from this cohort will be used to facilitate advanced and accurate diagnostic techniques related to NCDs while considering various phenotypes. Potential collaborators can access the dataset after receiving approval from our institutional review board. Applications can be submitted on the study homepage (http://en-healthcare.snuh.org/HPEACEstudy).
Strengths and limitations of this study

- The strengths of the Health and Prevention Enhancement study include a large number of healthy subjects ( $n=91336$ healthy examinees, from the Healthcare System Gangnam Center, Seoul National University Hospital, between 2003 and 2014) and a structured and organised database.

- This study not only includes data widely used in medical check-ups but also data from sophisticated high-quality advanced examinations to investigate clinical effectiveness in predicting the predisease stage of non-communicable diseases, including malignancies and metabolic diseases, in an average-risk population in Korea.

- Another strength includes the active and passive follow-ups and the ability to obtain complete data, including deaths and incidental cancer cases, even among those who discontinued visiting our centre.

- The data from this study will allow us to contribute to active, effective prevention of the development of cancers and non-communicable diseases.

- The major weakness of this cohort is that it may show selection bias because only subjects who voluntarily visited our centre were included in the study.

\section{INTRODUCTION}

In recent decades, the prevalence of non-communicable diseases (NCDs), such as malignancy, metabolic disease and cardiovascular disease, has rapidly increased in Korea. ${ }^{12}$ To address this problem, a comprehensive approach that accounts for lifestyle, environmental factors and genetic variability is needed as NCDs are known to be caused by both genetic and environmental factors. ${ }^{34}$ Precision medicine is emerging as a potential solution. ${ }^{56}$ This type of medicine categorises individuals into different subgroups based on their susceptibility to disease and then focuses 
on individuals in whom interventions will be helpful. ${ }^{7}$ One of the major components of efficient prevention and early detection of NCDs is thus identifying high-risk populations among those in a predisease or asymptomatic stage.

The Seoul National University Hospital (SNUH) Healthcare System Gangnam Center provides comprehensive medical check-ups and screening, and nearly 20000 people visit this centre each year. A data warehouse, HEALTH-WATCH, was built as a prototype database for this cohort study within the healthcare research institute from the start of the centre in $2003 .{ }^{8}$ Using HEALTH-WATCH, we have published many articles that have primarily been focused on cancer screening and metabolic diseases. ${ }^{8-19}$ In 2013, we reorganised the cohorts to support our research and started collecting blood samples to analyse the genetic factors involved in NCDs. We then summarised and integrated our clinical and genetic data in the Health and Prevention Enhancement (H-PEACE) study.

The H-PEACE study was designed to investigate the predisease stage of NCDs, which helps us assess an individual's risk of NCDs and establish personalised prevention strategies. Through analyses of longitudinal data from comprehensive questionnaires and clinical and laboratory tests, the H-PEACE study can expand our knowledge of NCD prevention, as well as our knowledge of high-risk populations, to improve the early detection of NCDs. The findings can inform treatment priorities and therapeutic guidelines related to NCDs. Finally, the H-PEACE study can contribute to enhancing public health and improving quality of life.

\section{COHORT DESCRIPTION \\ Participants}

The H-PEACE study collected data from 91336 Koreans aged $45.5 \pm 11.7$ years (50507 men and 40829 women) who received a health check-up between October 2003 and December 2014 at the SNUH Gangnam Center (IRB no. H-1311-031-531). We prospectively collected blood samples from 17455 of those individuals (9396 men and 8059 women) from October 2013 to form the Gene-environmental interaction and phenotype (GENIE) study, a subcohort of the H-PEACE study. Furthermore, we obtained data for genome-wide single-nucleotide polymorphism (SNP) arrays using 6579 donated blood samples. The flow chart of the H-PEACE study subjects is illustrated in figure 1 , and the distribution of the participants in Korea in figure 2.

\section{Repeated measurement and active and passive follow-up}

Annual health exams are mandatory for all workers under the Industrial and Safety Law in Korea. In the H-PEACE study, there are two follow-up systems (passive and active follow-up system) for tracking the development of the new diseases and death of the participants. We have annually updated the survival data by linking to National
Death Certificates through requests to Statistics Korea. Under the passive follow-up systems, $>99 \%$ of participants are followed up for their new cancer development and death annually.

Moreover, the study participants are followed up for their new disease status by revisit to centre for repeated measurement and assessment system. This active follow-up system allows the subject to voluntarily visit our centre. The health check-up system in Korea has two systems in which the National Health Insurance Corporation (NHIC) pays for participants' basic health examination fee once every two years or in which the health examination participants pay all expenses for their health examination fees. The latter programme includes more precise health examination testing that individuals want, and all costs must be paid by individuals. We are under the latter system, and the health examination participants have to pay for all the health check-ups under their voluntary visit. The participants who have undergone health examinations at our centre may receive health check-ups afterwards at different centres that are under partial coverage of the NHIC without revisiting our centre. Although the health check-up services conducted in our centre include a variety of tests that the subject wants, including the basic health check-up programme conducted by the NHIC, we have to make a lot of effort in order for the subject to return to our centre because the former health check-up system covered by the NHIC is free-paid for the basic health check-up programme. We are using a number of ways to encourage their return to our centre. To encourage participants, we provide reminder calls every year and send healthcare information about people's next health check-up date via phone and letter. So the eligibility for follow-up assessment depends on the participants' voluntary revisit need and self-payment availability.

Under this active follow-up system, we did a repeated measurement of risk factors and an assessment of outcome variables for 46484 individuals $(50.9 \%)$ of total 91336 cohort members who participated in the baseline health check-up once within 4 years from the first visit and 74304 individuals $(83.5 \%)$ were actively followed up once within 10 years from the first visit. Moreover, 50049 participants $(54.8 \%)$ completed the two or three repeated measurements (figure 1 ). The median follow-up was 4.04 years (IQR 2.1-6.5).

Of 91336 cohort members, a total of 17455 members (19.1\%) agreed to blood collection and had several aliquots of blood specimens. Of 17455 members, 6579 participants were included in the GENIE study with genome-wide association study (GWAS) information $(37.7 \%)$, and of them, 5915 participants $(89.9 \%)$ completed at least first follow-up test within 4 years of the first visit. The median follow-up of the GENIE study was 5.23 years (IQR 2.7-8.0).

\section{Data collection}

The H-PEACE study consists of core and specific variables (tables 1 and 2). The core variables were tests performed 


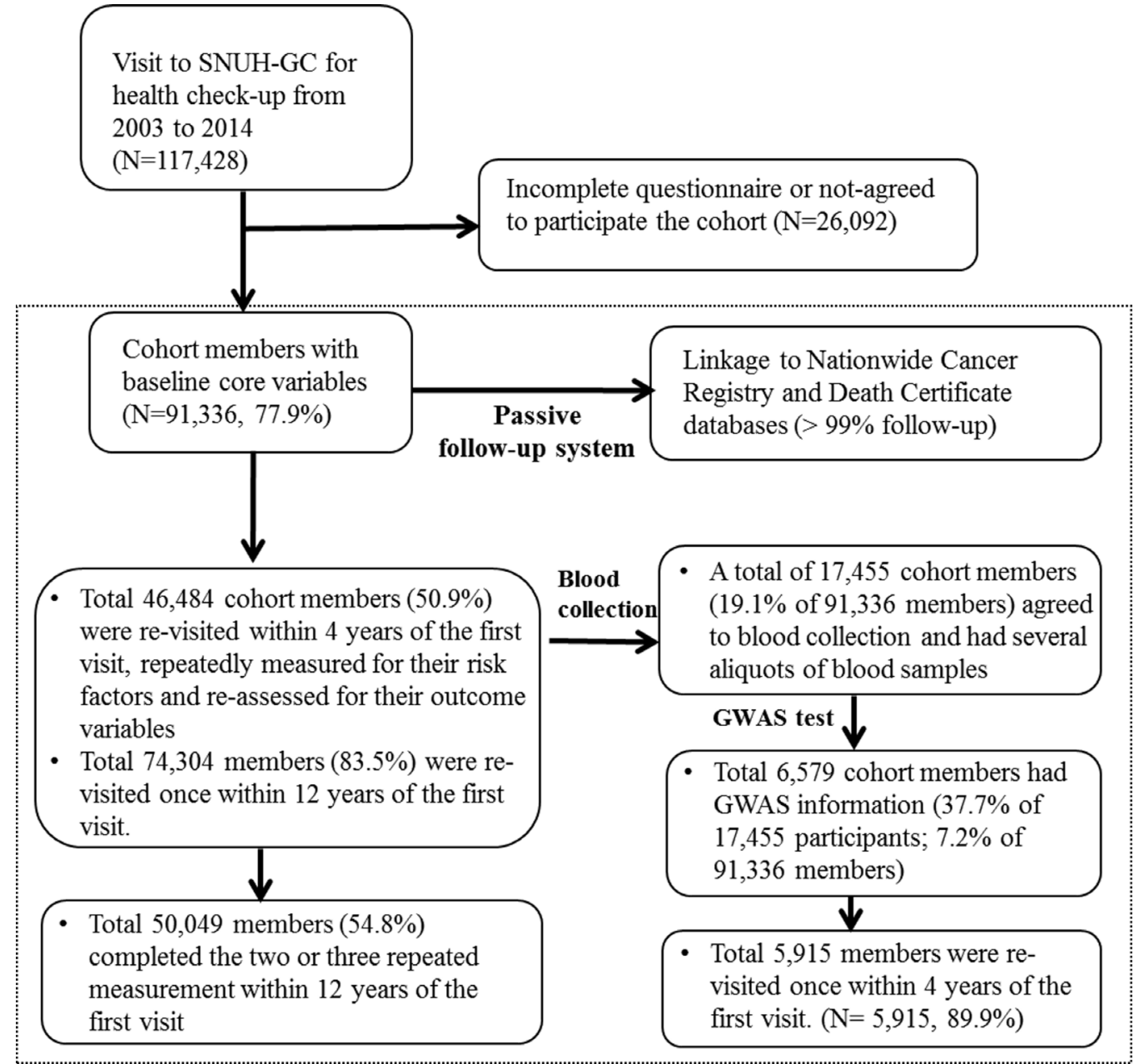

Figure 1 Study flow diagram of the Health and Prevention Enhancement study.GC, Gangnam Center; GWAS, genome-wide association study; SNUH, Seoul National University Hospital.

in all of the enrolled participants at the regular health check-ups. The specific variables were the tests selectively performed in the participants on request or based on the recommendation of their medical provider according to the participant's symptoms or disease risk factors. These tests were sophisticated and expensive tests that are rarely used in other cohort profiles. In table 3 , we describe the representative test for each variable and its participation rate. The tests performed for the covariables and specific variables are described below. All data were collected as part of the comprehensive health check-ups. The flow diagram of health check-ups in our study is illustrated in figure 3. All study protocols are available on request at our homepage (http://en-healthcare.snuh.org/ HPEACEstudy).

\section{Structured questionnaires}

Self-reported questionnaires were used to obtain socio-demographic data, personal and familial medical history, health-related behaviours (such as smoking status, alcohol consumption, physical activity, dietary behaviour), International Prostate Symptom Score, female reproductive factors and psychological status. Physical activity levels were assessed using the validated Korean version of the International Physical Activity Questionnaire short form and were classified into three categories: inactive, minimally active and health-enhancing physically active (HEPA). ${ }^{20} 21$ Dietary habits were evaluated using the Mini-Dietary Assessment Index, which was validated in Korean. ${ }^{22}$ Psychological status was assessed by the Beck Depression Inventory or Quick Inventory of Depressive Symptomatology-Self Report. ${ }^{23}$

\section{Physical examinations}

Blood pressure was measured using sphygmomanometers with patients in a seated position after a resting period. If systolic blood pressure was $\geq 140 \mathrm{~mm} \mathrm{Hg}$ or diastolic blood pressure was $\geq 90 \mathrm{~mm} \mathrm{Hg}$ after a rest period and two measurements, we recorded the values and calculated their averages. Height $(\mathrm{cm})$, weight $(\mathrm{kg})$, waist circumference $(\mathrm{cm})$ and body fat composition $(\%)$ were measured by trained nurses with participants wearing a lightweight hospital gown and in bare feet. Height and weight were measured using digital scales in a standing position. Waist circumference was obtained by measuring the smallest natural waist circumference area, which is around the umbilicus using a non-stretch tape measure, without any pressure to the body surface during measurements. 


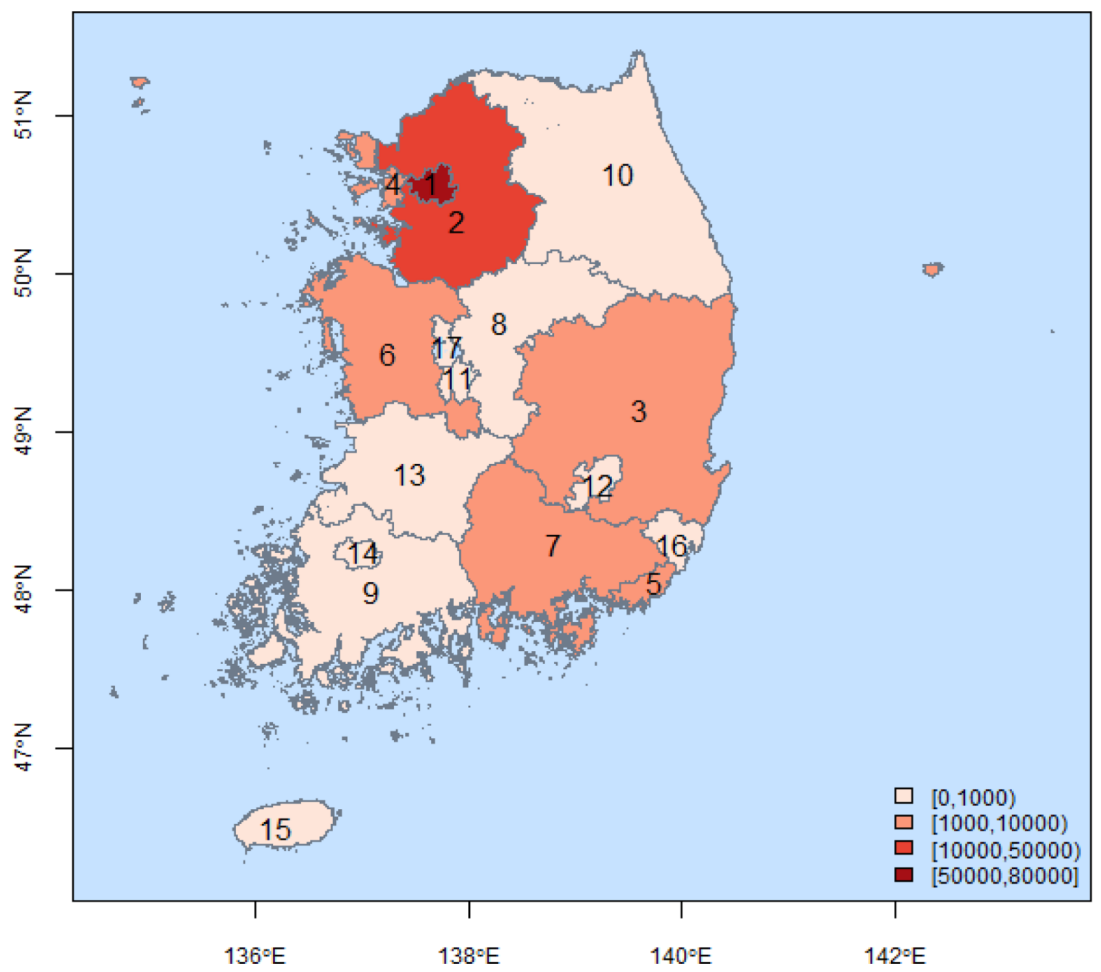

\begin{tabular}{llc}
\hline & \multicolumn{1}{c}{ Location } & $\mathrm{N}$ \\
\hline 1 & Seoul (Capital, Metropolitan) & 60,301 \\
2 & Gyeonggi-do & 18,383 \\
3 & Gyeongsangbuk-do & 1,564 \\
4 & Incheon (Metropolitan) & 1,478 \\
5 & Busan (Metropolitan) & 1,445 \\
6 & Chungcheongnam-do & 1,400 \\
7 & Gyeongsangnam-do & 1,278 \\
8 & Chungcheongbuk-do & 769 \\
9 & Jeollanam-do & 737 \\
10 & Gangwon-do & 727 \\
11 & Daejeon (Metropolitan) & 692 \\
12 & Daegu (Metropolitan) & 686 \\
13 & Jeollabuk-do & 580 \\
14 & Gwangju (Metropolitan) & 488 \\
15 & Jeju-do & 338 \\
16 & Ulsan (Metropolitan) & 297 \\
17 & Sejong (Metropolitan) & 20 \\
\hline & $\quad$ Total & 91,183 \\
\hline
\end{tabular}

Figure 2 Regional distribution of the Health and Prevention Enhancement study in Korea.

Percentage of body fat and visceral fat area were estimated with a multifrequency bioimpedance analyser with eight-point tactile electrodes (Inbody 720, Biospace, Seoul, Korea). Comprehensive eye examinations (visual acuity, ocular tonometry, slit-lamp test, fundus photography) and hearing tests were also performed.

\section{Laboratory tests}

Blood samples from the antecubital vein were collected after at least 10 hours of fasting. The blood parameters assessed included complete blood cell count, fasting blood glucose, glycated haemoglobin, uric acid, blood urea nitrogen, creatinine, total calcium, inorganic phosphorus, glucose, sodium, potassium, chloride, total $\mathrm{CO}_{2}$, total protein, albumin, total bilirubin, aspartate transaminase, alanine transaminase, gamma-glutamyl transferase , total cholesterol, low-density lipid cholesterol, high-density lipid cholesterol, triglycerides, high-sensitivity $\mathrm{C}$ reactive protein concentration, prothrombin time, activated partial thromboplastin time, hepatitis B surface antigen, anti-HBs, HAV Ab IgG, alpha-fetoprotein, cancer antigen 19-9, carcinoembryonic antigen and prostate-specific antigen in men and cancer antigen 125 in women. The laboratory medicine department at the SNUH has been certified by the Korean Society of Laboratory Medicine and participated in the College of American Pathologist's Survey/Proficiency Testing programme. Urine tests were performed by stick test using spot urine. Semiquantitative variables included $\mathrm{pH}$, protein, glucose, ketone, bilirubin and blood. Stool samples were collected to conduct faecal occult blood tests and parasite assays.

\section{Digital imaging and specific diagnostic variables}

Chest X-ray was included as a core variable of the H-PEACE study, which included specific information on diagnostic variables (table 1). First, the GENIE study collected donated blood samples from 17455 recipients, and genome-wide SNP arrays from 6579 donated blood samples have already been analysed using the Affymetrix platform (Axiom Customized Genome-Wide Human Assay) (table 3). We plan to increase the available genetic information by 2020 using an SNP array. The PLINK program V.1.9 and R statistics V.3.3.0 were used for quality control procedures and data analysis. SNP genotype data combined with clinical data from the H-PEACE study were used to evaluate gene-environment interactions and to define the related phenotypes.

In total, 12846 participants underwent coronary CT with a coronary calcium score to assess coronary calcification. The calcium score of the coronary artery is a strong predictor of myocardial infarction and sudden cardiac death. ${ }^{24}$ In addition, 34771 participants underwent visceral fat CT to measure visceral adipose tissue (VAT) and subcutaneous adipose tissue (SAT). The detailed methods used to measure VAT area and SAT area on abdominal fat CT images have been described elsewhere. ${ }^{17}$ This quantitative assessment of intra-abdominal adipose tissue is considered the gold standard for measuring the amount of visceral fat. ${ }^{25}$ With advanced imaging techniques including echocardiography, brain MRI/magnetic resonance angiogram, carotid Doppler ultrasound, abdominal ultrasonography and abdominal CT, we elucidated the correlation of visceral obesity 
Table 3 Participation rate for the core and specific variables collected in the Health and Prevention Enhancement (H-PEACE) study at baseline

\begin{tabular}{|c|c|c|}
\hline Variables & $\begin{array}{l}\text { Study } \\
\text { participants } \\
\text { (N) }\end{array}$ & $\begin{array}{l}\text { Participation } \\
\text { rate } \\
(\%)^{\star}\end{array}$ \\
\hline \multicolumn{3}{|l|}{ Core variables } \\
\hline Most core variables & $\geq 90377$ & $\geq 99$ \\
\hline ECG & 91197 & 99.8 \\
\hline Estimated GFR & 91197 & 99.8 \\
\hline Stool parasite examination & 82493 & 90.3 \\
\hline Helicobacter pylori lgG Ab & 81142 & 88.8 \\
\hline Thyroid function test & 77313 & 84.6 \\
\hline $\begin{array}{l}\text { CLO test or UBT test for } \\
\text { current } H \text { pylori infection }\end{array}$ & 67986 & 74.4 \\
\hline $\begin{array}{l}\text { Gastroendoscopy with } \\
\text { pathological report }\end{array}$ & 66451 & 72.8 \\
\hline
\end{tabular}

Specific variables

\begin{tabular}{|c|c|c|}
\hline Dental examination & 43232 & 47.3 \\
\hline $\begin{array}{l}\text { Colonoscopy with } \\
\text { pathological reports }\end{array}$ & 46050 & 50.4 \\
\hline $\begin{array}{l}\text { Abdomen CT with visceral } \\
\text { fat CT }\end{array}$ & 34771 & 38.1 \\
\hline Abdomen sonography & 33446 & 36.6 \\
\hline Echocardiography & 20688 & 22.7 \\
\hline Coronary CT & 12846 & 14.1 \\
\hline $\begin{array}{l}\text { Echocardiography, treadmill } \\
\text { test }\end{array}$ & 9910 & 10.9 \\
\hline SNP from GWAS & 6579 & 7.2 \\
\hline $\begin{array}{l}\text { Bone densitometry } \\
\text { (postmenopausal females) }\end{array}$ & 26376 & 99.4 \\
\hline $\begin{array}{l}\text { E2 levels (postmenopausal } \\
\text { females after } 5 \text { years from } \\
\text { menopause) }\end{array}$ & 4742 & 49.9 \\
\hline $\begin{array}{l}\text { Bone densitometry } \\
\text { (men } \geq 50 \text { years) }\end{array}$ & 9488 & 48.9 \\
\hline T levels (men $\geq 60$ years) & 6697 & 52.9 \\
\hline HPV genotyping (females) & 12999 & 31.8 \\
\hline Pap smear (women $\geq 35$ years) & 12951 & 49.1 \\
\hline $\begin{array}{l}\text { Blood collection for the GENIE } \\
\text { study }\end{array}$ & 17455 & 19.1 \\
\hline SNP variations in the GWAS & 6579 & $37.7 \dagger$ \\
\hline
\end{tabular}

*Participation rate of all 91336 H-PEACE cohort members. †Participation rate of all 17455 GENIE study members (a subcohort of the H-PEACE study).

CLO, Campylobacter-like organism; GENIE, Geneenvironmental interaction and phenotype; GFR, glomerular filtration rate; GWAS, genome-wide association study; HPV, human papillomavirus; SNP, single-nucleotide polymorphism; UBT, urea breath test.

with vascular disease using both data in a complementary manner. The core variables of the H-PEACE study including the blood test results, questionnaire findings and depression scores can contribute to determining the correlation between visceral obesity and metabolic phenotype.

We collected ECG reports from 91197 consecutive recipients to evaluate the incidence and risk factors of atrial fibrillation, a significant risk factor for stroke. The effects of a bundle branch block or atrioventricular block on stroke and cardiac disease were also analysed. Furthermore, we collected Holter monitor results to confirm the ECG reports.

Human papillomavirus (HPV) is known to lead to cervical cancer in women. ${ }^{26}$ Of the participants in the H-PEACE study, 12951 women underwent both a liquidbased cervical cytology (SurePath LBC, Becton Dickinson, Franklin Lakes, New Jersey, USA) and an HPV genotyping test using an HPV DNA chip (MyHPV Chip, Biomedlab, Seoul, Korea) for cervical cancer screening. ${ }^{27}$ We also included the results of gynaecological sonography, as well as venereal disease research laboratory and HIV tests.

WHO considers Helicobacter pylori infection a class I carcinogen for gastric cancer. ${ }^{13}$ The diagnosis of $H$. pylori infection was based on the detection of serum $H$. pylori IgG antibody using a kit (H. pylori-EIA-Well, Radim, Rome, Italy) that was previously validated in a nationwide Korean seroepidemiological study. ${ }^{28}$ Furthermore, 66451 recipients had available upper endoscopy data with pathology results. Serum pepsinogen data and eradication history were also collected.

A total of 46050 participants in the H-PEACE study received a colonoscopy in our centre. All colonoscopies were conducted by board-certified endoscopists, and the average adenoma detection rate in recipients aged 50-70 years was $>30 \% .{ }^{12}$ Endoscopes including the CF-H260 and CF-HQ290 series (Olympus, AIZU, Japan) and the EC-450HL5, EC-450WM5 and EC-590ZW series (Fujinon, Saitama, Japan) were used. Histological diagnoses at our centre were determined according to the WHO classification of tumours of the digestive system. ${ }^{29}{ }^{30}$ All colonoscopy results and corresponding pathology reports were collected.

We calculated estimated glomerular filtration rate (GFR) using the chronic kidney disease epidemiology collaboration equation and the modification of diet in renal disease formula. We collected GFR data successively to identify the risk factors for chronic kidney disease. We also included spot urine sodium and creatinine ratio and 24-hour urine data including GFR.

Dental exams were conducted in 43232 participants. Periodontitis is highly prevalent among adults and is one of the most common causes of teeth loss after 40 years. ${ }^{31-33}$ Furthermore, periodontitis can induce systemic inflammation, as well as masticatory dysfunction and poor nutritional status. ${ }^{32}$ This is the first cohort study to include a large number of dental exams to evaluate the progression of periodontitis. In this study, 43232 participants received a dental exam; $33.1 \%$ had mild to moderate periodontitis and $2.1 \%$ had severe periodontitis. We also included participants' colonoscopy and coronary CT results, including coronary calcification and colon polyps, to 


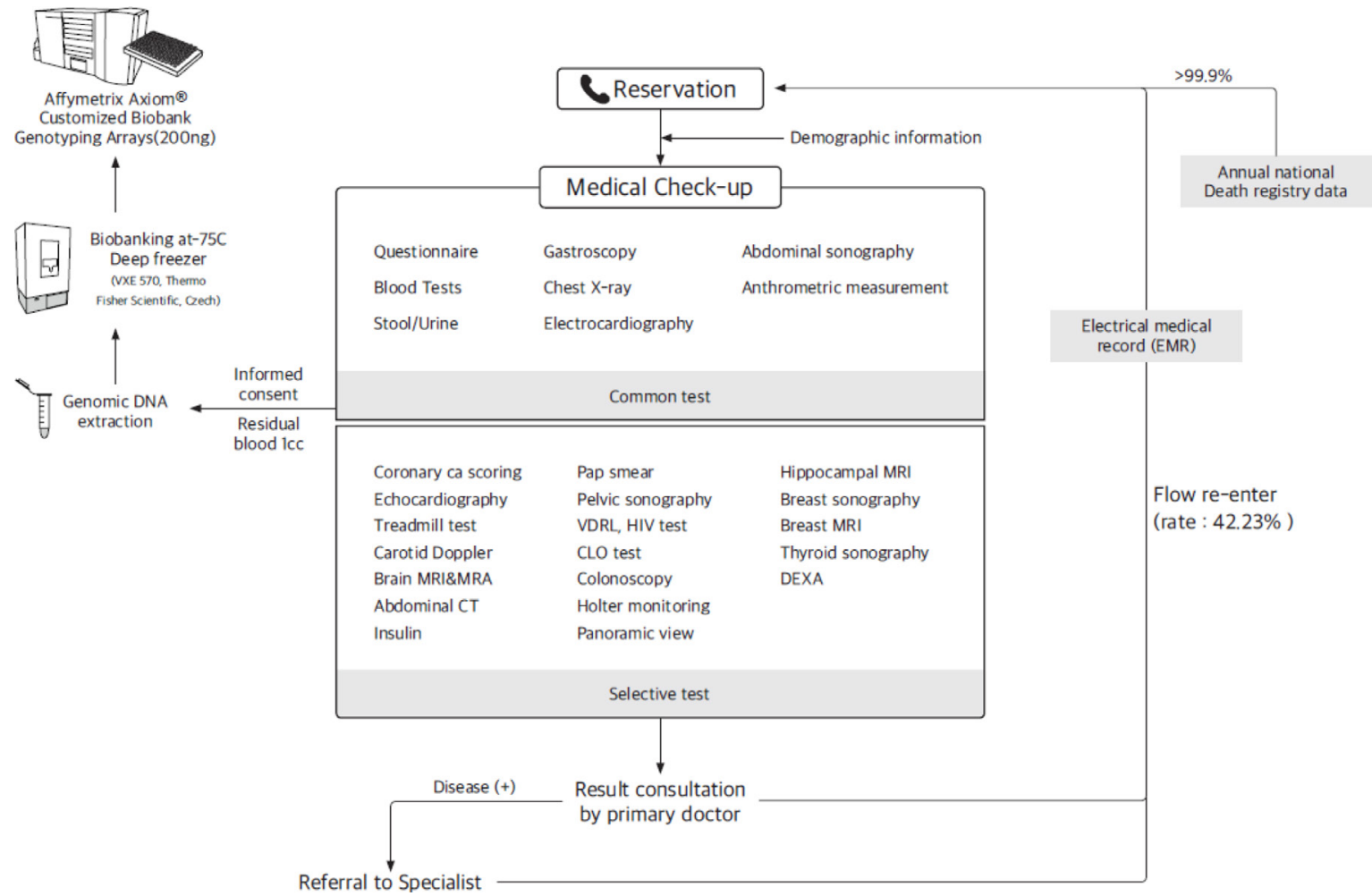

Figure 3 Flow diagram of health check-ups in the Health and Prevention Enhancement study. CLO, Campylobacter-like organism; DEXA, dual-energy X-ray absorptiometry; MRA, magnetic resonance angiogram; VDRL, venereal disease research laboratory

assess the correlation between periodontitis and systemic inflammation.

The presence of osteoporosis was determined using bone densitometry in 26207 women and 9488 men. We measured bone mineral density (BMD) of the lumbar spine (L1-L4) and femur using dual-energy X-ray absorptiometry (GE Medical, UK). Based on participants' lowest T scores, normal BMD, osteopenia and osteoporosis were defined as $\mathrm{T}$ scores of $-1.0 \mathrm{SD}$ and above, -1.0 to -2.5 SDs and -2.5 SDs and below, respectively. In this cohort, $26.6 \%$ of the participants who underwent bone densitometry were male. Of those, osteopenia was found in $24.75 \%$ and osteoporosis was found in $3.21 \%$. The information collected in this cohort also included hormone levels, oestrogen levels in women and testosterone levels in men.

\section{FINDINGS TO DATE}

The baseline characteristics are summarised in table 4, and the morbidity rate of the major outcomes is shown in table 5. The morbidity rates were calculated as the number of findings divided by the number of participants tested. The prevalence of major outcomes (mortality and cancer incidence rate) is provided in table 6 . The H-PEACE study has contributed to the research community by publishing $>200$ articles since the initiation of the data warehouse, HEALTH-WATCH, as a prototype in $2003 .{ }^{8}$ Our studies have primarily focused on cancer screening and identifying the risk factors and prognosis of metabolic disease.
For subjects with 1-2 adenomas $<10 \mathrm{~mm}$ in colonoscopy for colon cancer screening, they were classified as a low-risk group having low 5-year colon adenoma incidence rates and were recommended to take further colonoscopic screening test after 5 years. For subjects with advanced adenoma, multiple adenoma $\geq 3$ or larger adenoma sized $\geq 10 \mathrm{~mm}$ in baseline colonoscopy for colon cancer screening, they were classified as a high-risk group having higher incidence rates of advanced adenoma or higher recurrence rates of adenoma and were recommended to take colonoscopic surveillance at 3 years after initial polypectomy. ${ }^{12}$ This strategy of colonoscopic surveillance has been reflected in the guidelines for colonoscopic surveillance. ${ }^{34}$ In our study, the colonic adenoma incidence rate was $37 \%$. Our centre performed a previous study on the prevalence and risks of colorectal adenoma in asymptomatic Koreans aged 40-49 years undergoing screening colonoscopies. ${ }^{35}$ In that study, the prevalence of adenoma was $22.2 \%$ in the age group of $40-49$ years and $32.8 \%$ in the age group of $50-59$ years. This finding is quite consistent with that in our cohort. In the paper, we concluded that the prevalence of adenoma in subjects aged 40-49 years was higher than that in previous studies. Male sex and current smoking habits were among the risk factors.

We also examined the quality of colon cancer screening using metrics such as the adenoma detection rate. ${ }^{36}$ Additionally, we evaluated the influence of image-enhanced endoscopy, such as narrow band imaging and Fuginon Intelligent Color Enhancement, using our cohort 
Table 4 Baseline characteristics of the participants in the Health and Prevention Enhancement study

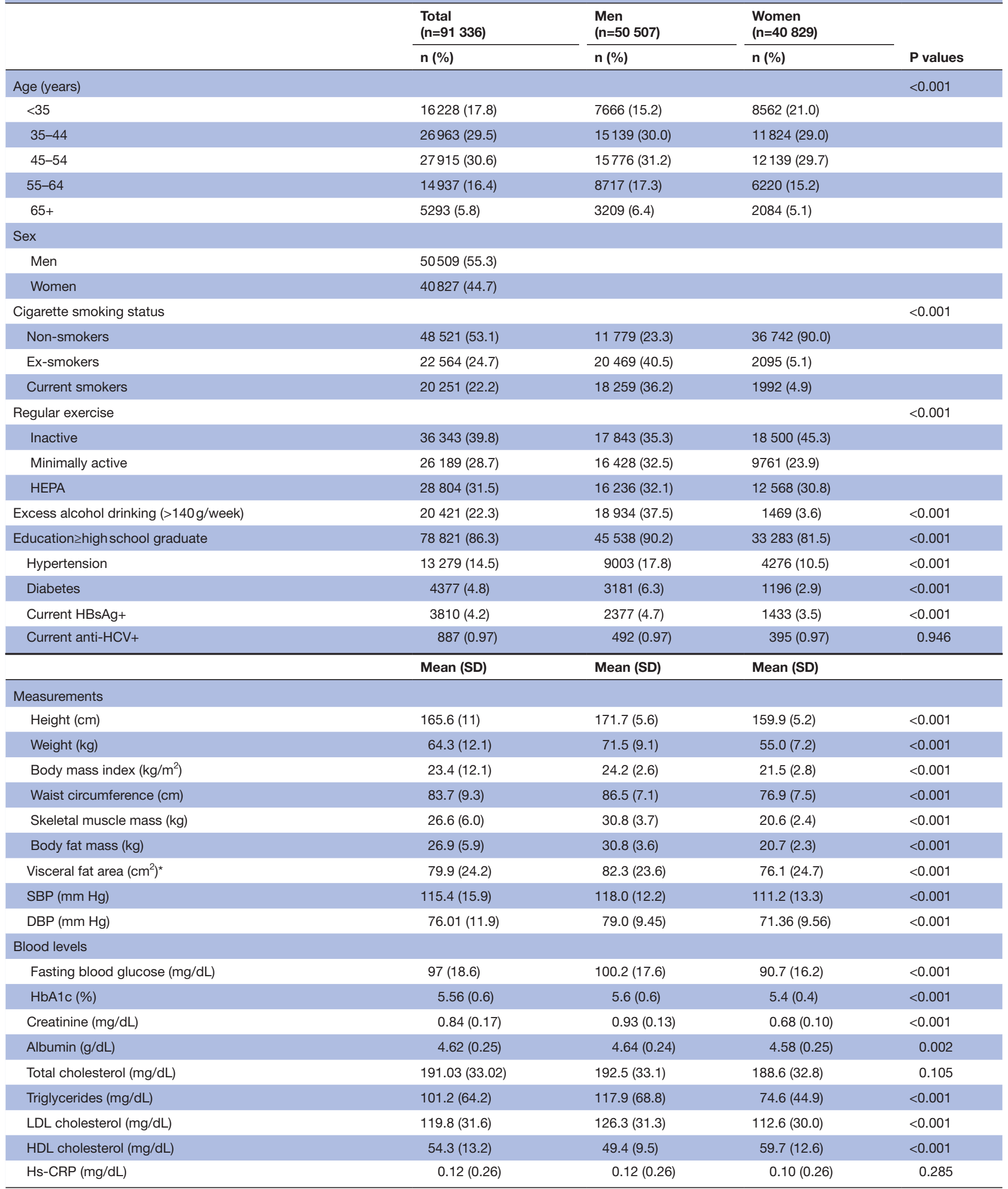

*Visceral fat area is defined here as the cross-sectional area of visceral fat found in the abdomen.

Anti-HCV, anti-hepatitis C antibody; DBP, diastolic blood pressure; HBsAg, hepatitis B surface antigen; HDL, high-density lipid; HEPA, health-enhancing physically active; Hs-CRP, high-sensitivity C reactive protein; LDL, low-density lipid; SBP, systolic blood pressure. 
Table 5 Morbidity rates after active follow-up with specific measurements in the Health and Prevention Enhancement study

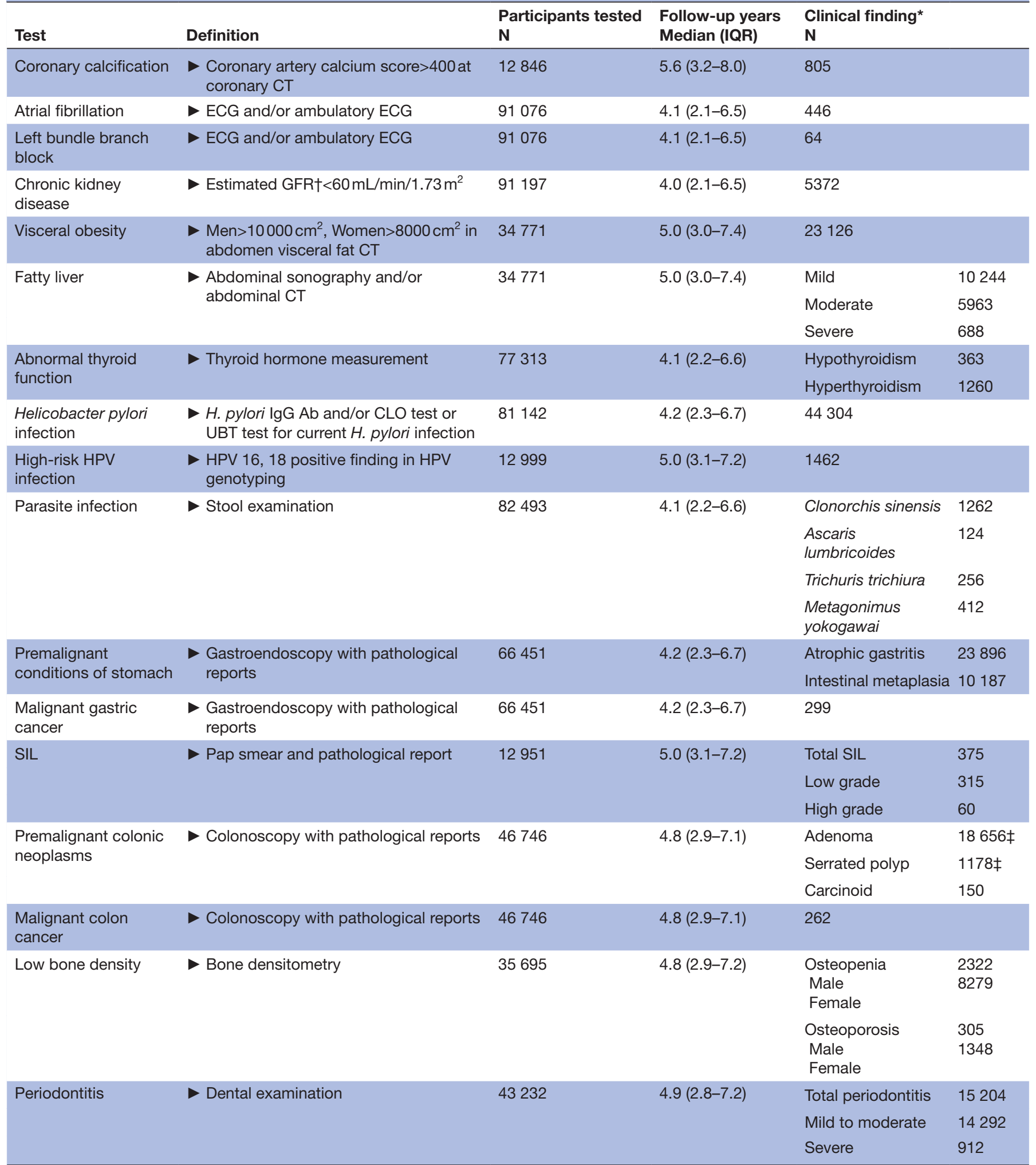

${ }^{*}$ The number of findings among the participants who were at risk.

†The GFR was estimated by the Chronic Kidney Disease Epidemiology Collaboration equation and the Modification of Diet in Renal Disease formula.

$\ddagger$ Adenoma and serrated polyp detection rate were calculated in participants aged $50-75$ years.

CLO, Campylobacter-like organism; GFR, glomerular filtration rate; HPV, human papillomavirus; SIL, squamous intraepithelial lesion; UBT, urea breath test. 
Table 6 Major cancer incidence and outcomes based on record linkage to the Nationwide Death Certificate Database in the Health and Prevention Enhancement (H-PEACE) study*

\begin{tabular}{|c|c|c|c|c|c|c|}
\hline & \multicolumn{3}{|c|}{ Cancer incidence (n) } & \multicolumn{3}{|c|}{ All-cause death† (n) } \\
\hline & Total & Men & Women & Total & Men & Women \\
\hline Total cancer & 2814 & 1402 & 1412 & 207 & 150 & 57 \\
\hline Thyroid & 956 & 390 & 566 & 3 & 3 & 0 \\
\hline Stomach & 513 & 337 & 176 & 37 & 29 & 8 \\
\hline Breast & 343 & 1 & 342 & 6 & 0 & 6 \\
\hline Colorectal & 284 & 182 & 102 & 28 & 20 & 8 \\
\hline Lung & 186 & 109 & 77 & 45 & 32 & 13 \\
\hline Prostate & 127 & 127 & 0 & 3 & 3 & 0 \\
\hline Kidney & 91 & 71 & 20 & 1 & 1 & 0 \\
\hline Liver & 87 & 71 & 16 & 31 & 28 & 3 \\
\hline Gynaecology & 52 & 0 & 52 & 7 & 0 & 7 \\
\hline Pancreas & 42 & 25 & 17 & 27 & 19 & 8 \\
\hline Brain & 39 & 20 & 19 & 0 & 0 & 0 \\
\hline Haematological & 39 & 24 & 15 & 5 & 3 & 2 \\
\hline Biliary tract & 32 & 26 & 6 & 11 & 9 & 2 \\
\hline Oesophagus & 23 & 19 & 4 & 3 & 3 & 0 \\
\hline
\end{tabular}

${ }^{*}$ Median (IQR) follow-up years of the 91336 participants=4.04 (2.13-6.5).

†Since the Nationwide Death Certificate Database does not provide information about cause of death, number of death includes death from not only cancer but also other causes. Among the $91336 \mathrm{H}$-PEACE participants, the all-cause mortality rate was $969: 715$ men and 254 women.

data. ${ }^{10} 3738$ For gastric cancer screening, the effect of screening endoscopy remains controversial, but population-based screening has been undertaken in Korea and Japan. For participants with intestinal metaplasia in gastroendoscopy, we also classified them into highrisk group and recommended an annual endoscopic screening, based on study results that people with strong risk factors such as male and an older age can quickly find early-staged endoscopically treatable gastric cancer by taking annual gastroendoscopic screening. ${ }^{11}$ In our cohort, $54 \%$ had H. pylori infection and $36 \%$ had atrophic gastritis. In a nationwide multicentre study in Korea performed by our centre for the prevalence of $H$. pylori infection, the seropositive rate of H. pylori was $59.6 \% .^{39}$ This rate is quite consistent with our study result and consequently explains the relatively high percentage of atrophic gastritis that we observed. Gastric mucosa-associated lymphoid tissue lymphomas (MALToma) and suspicious MALToma lesions were also detected in screening endoscopies, and the prevalence of gastric MALToma was high in middle-aged women. ${ }^{40}$ Active endoscopic screening for gastric cancer had the additional benefit of detecting early-stage MALToma. ${ }^{40}{ }^{41}$ Furthermore, we established that small subepithelial tumours that were incidentally found during upper endoscopy screening showed no size change in subsequent follow-up periods. ${ }^{37}$

Our research focus was not limited to cancer screening. We also examined metabolic diseases such as non-alcoholic fatty liver disease (NAFLD) and metabolic syndrome. Consistent with our results, the population prevalence of NAFLD in Korea is $>25 \%$, like that in many Western countries. ${ }^{19-42}$ Although subjects in this cohort are relatively young and lean, lean NAFLD (or non-obese NAFLD) is more common in Asians (including Koreans) than in Western populations. Asians generally have a higher percentage of visceral fat and body fat than people of other races of the same age, sex and even body mass index (BMI) ${ }^{45-47}$ As visceral obesity is a major risk factor for lean NAFLD, this might have affected the higher prevalence of NAFLD in this cohort, comprising relatively lean subjects. ${ }^{45}$ In addition, the term 'fatty liver' in this paper includes not only NAFLD but also alcoholic fatty liver disease. The proportion of subjects who drink alcohol heavily in this cohort is as high as $37.5 \%$ in men, which might affect the high prevalence of fatty livers that we observe.

We reported that metabolic syndrome was an independent risk factor of silent brain infarction using brain MRI data (OR 2.18; 95\% CI 1.38 to $5.82, \mathrm{p}=0.001$ ). ${ }^{48}$ The prevalences of metabolic syndrome and its components were higher in subjects with silent brain infarctions than in those without. This is the first study to demonstrate an association between metabolic syndrome and silent brain infarction. This finding might help identify healthy people at increased risk for developing silent brain infarction. We also found an effect of body fat distribution on the incidence of NAFLD and reflux oesophagitis using abdominal fat CT data. ${ }^{9}{ }^{14}$ We identified a relationship between NAFLD and the risks of coronary heart disease and arterial stiffness based on coronary CT and the 
cardio-ankle vascular index (CAVI), respectively. ${ }^{16-19}$ To elucidate the relationship between NAFLD and the risk of coronary heart disease, we used the coronary artery calcification score as measured by coronary CT. This measurement showed that patients with NAFLD are at increased risk for coronary atherosclerosis (OR 1.28, 95\% CI 1.04 to $1.59, \mathrm{p}=0.023) .{ }^{17}$ In the study on the association between NAFLD and arterial stiffness, we used CAVI, a new measurement of arterial stiffness. In an age-adjusted, sex-adjusted and BMI-adjusted model, NAFLD was associated with a $42 \%$ increase in the risk for arterial stiffness, and this increased according to the severity of NAFLD. ${ }^{16}$ The effect of physical activity on NAFLD was also demonstrated using data from the questionnaires. ${ }^{49}$

The list and number of publications can be requested on our homepage (http://en-healthcare.snuh.org/ HPEACEstudy).

\section{STRENGTHS AND LIMITATIONS}

This study has several potential limitations as below. First, our study participants are composed of individuals who voluntarily visited our centre and their data collection for the repeated measurements rely on participants' selfpaid. Our system of enrolling cohort members includes a potential selection bias. Second, self-recorded questionnaires are used to obtain the information of risk factors and disease histories before the next visit of study participants to our centre, and this procedure leads to recall bias and response bias. Third, our active follow-up rates in assessing outcome variables and repeatedly measuring risk factors are not high, leading to selection bias. However, since active follow-up rates are increasing every year, at least $87 \%$ of follow-up is expected if all subjects are terminated for at least 10-year follow-up. Forth, in our cohort, we used to measure waist circumference as the definition of the smallest area around the belly button below the rib cage and above the hip bone, although the ideal way is to measure the midway between the top of the hip bone and below the rib cage. The former is usually called the natural waist, and we used this former definition for practical reason to reduce intraindividual measurement bias in waist circumference. In health check-up at our centre, a lot of participants (about 100-120 health examinees) every day visit the centre for health examination and many health technicians and nurses take measurement of waist and hip circumferences. For each participant, there is a very little chance to take measurement of waist at the time of revisit by same nurses met at the time of cohort enrolment. The intraindividual measurement bias in measuring waist circumference may be problematic at our centre and thus we thought that it was necessary to use the most practical and easy waist measurement together with repeated nurse training. We did a small pilot study for measuring waist by the two methods for 10 men and 16 women. The ideal measuring method (midway measurement) has a limit in consuming time and effort due to difficulty in method itself. In spite of short time and smaller effort in measuring waist, the natural waist measuring method showed an excellent agreement (intraclass correlation coefficients $=99 \%$ in men and $93 \%$ in women) with ideal method and there were no shift between obesity groups classified by each method. Fifth, there is no information of cancer histology subtypes for cohort participants ascertained as new cancer development. Individual cancer development in study participants was ascertained with data linkage to the nationwide cancer registry data. Korea cancer registry data include International Classification of Diseases, Tenth Revision code, T-code (Topography) and M-code (Morphology in primary cancer sites). For example, among the stomach cancer types, $51.3 \%$ were adenocarcinomas, $22.1 \%$ were tubular adenocarcinomas and only $4.9 \%$ were histological NOS. ${ }^{50}$ Future studies will attempt to merge the individual data associations of M-code and T-code. Finally, the definition of a disease status could be changed during the follow-up periods as new guidelines are declared. For instance, guideline for the Prevention, Detection, Evaluation, and Management of High Blood Pressure in Adults opened at November $2017^{51}$ recommending a new definition for hypertension. This paper was written before the declaration was done. We have the raw data of blood pressure and the definition can be applied to the study population. But for those who had already been under antihypertensive medication at the point of enrolment in our cohort, they had followed the previous guidelines for indication of treatment. This makes it difficult to reclassify these patients according to the new guidelines. In the future follow-up period, we will pay close attention to clearly declare the definition of disease we are applying according to the period of terms.

A major strength of the H-PEACE study is its structured and organised database, which not only includes data widely used in medical check-ups but also data from sophisticated high-quality advanced examinations, such as colonoscopy, brain MRI, abdominal CT and abdominal fat CT. Particularly in our study, comprehensive biomarkers related to NCDs, including cancer antigens and infection markers, were included, which have not been included in the medical check-ups of other cohort profiles. It has only been about 10-15 years since the comprehensive health check-up programme was actively performed in Korea, and in the past decade, a large proportion of people participated in the check-up due to the support from the affiliated company's welfare policy. This might result in the relatively young population enrolment in our study population. But since the health check-up programme is getting more general, more elderly population is taking the health check-up. New enrolment is another future target study of our centre. Since the enrolled cohort population is relatively young, it is possible to study the preclinical disease stage and its final long-term outcomes. This might be the characteristics of our cohort that we could design a lot of prediction models for the NCD by using data from the preclinical stages. As we obtained a large sample size, we could confirm disease statuses as 
well as numerous phenotypes of NCDs. Furthermore, the study protocols were conducted and monitored with intensive quality control procedures. Additional strengths include the active and passive follow-ups and the ability to obtain complete data, covering deaths and incidental cancer cases even among those who discontinued visiting our centre. We believe that these data could contribute to active, effective prevention against the development of cancers and NCDs.

\section{Author affiliations}

${ }^{1}$ Healthcare Research Institute, Healthcare System Gangnam Center, Seoul National University Hospital, Seoul, South Korea

${ }^{2}$ Department of Preventive Medicine, Seoul National University College of Medicine, Seoul, South Korea

${ }^{3}$ Department of Biomedical Science, Seoul National University Graduate School, Seoul, South Korea

${ }^{4}$ Seoul National University Cancer Research Institute, Seoul, South Korea ${ }^{5}$ DNA link, Inc., Seoul, South Korea

Acknowledgements The authors thank Mi Kwon and Si Won Choi for their technical assistance and all the contributions of the H-PEACE study investigators.

Collaborators The genotype (SNP) data from the GENIE study and the epidemiological data from the other cohorts are available to researchers after a quality control process has been completed. Potential collaborators can access the dataset after receiving approval from the SNUH Gangnam Center institutional review board. Applications can be submitted via our homepage. Further information is available at the H-PEACE study website (http://en-healthcare.snuh.org/ HPEACEstudy).

Contributors CL, EKC, JMC, SKP, SJC, M-SK, J-EL, JSK and S-HC were involved in the conception and design of the study. CL, EKC, JMC, YH, YL, MK, JL, SJC and BP contributed to development of methods and data collection. CL, EKC, JMC, YL, SKP, $\mathrm{SC}$ and BP were involved in data analysis and interpretation. CL, EKC, JMC, SKP and SC drafted the manuscript. All the authors have critically revised the article and approved the final version and the findings.

Funding This work was supported by the SK Telecom Research Fund through Seoul National University Hospital (34-2013-0390 and 34-2014-0210 to SJC) and the Seoul National University Hospital Research Fund (04-2011-0700 to SJC).

Competing interests JEL is an employee of DNALink.

Patient consent Not required.

Ethics approval Study protocols were approved (IRB nos. H-1103-127-357 and H-1505-047-671).

Provenance and peer review Not commissioned; externally peer reviewed.

Data sharing statement Researchers can apply for the dataset after receiving approval from the SNUH Gangnam Center institutional review board. The H-PEACE study website provides information on the application process (http://en-healthcare. snuh.org/HPEACEstudy).

Open Access This is an Open Access article distributed in accordance with the Creative Commons Attribution Non Commercial (CC BY-NC 4.0) license, which permits others to distribute, remix, adapt, build upon this work non-commercially, and license their derivative works on different terms, provided the original work is properly cited and the use is non-commercial. See: http://creativecommons.org/ licenses/by-nc/4.0/

(C) Article author(s) (or their employer(s) unless otherwise stated in the text of the article) 2018. All rights reserved. No commercial use is permitted unless otherwise expressly granted.

\section{REFERENCES}

1. Beaglehole R, Bonita R, Horton R, et al. Priority actions for the noncommunicable disease crisis. Lancet 2011;377:1438-47.

2. Ministry of Health and Welfare of Korea, Korea Centers for Disease Control and Prevention. 2010 Korea Health statistics. Seoul: Ministry of Health and Welfare of Korea, 2011.
3. Hunter DJ. Gene-environment interactions in human diseases. Nat Rev Genet 2005;6:287-98.

4. Renz H, von Mutius E, Brandtzaeg P, et al. Gene-environment interactions in chronic inflammatory disease. Nat Immunol 2011:12:273-7.

5. Collins FS, Varmus H. A new initiative on precision medicine. N Engl $J$ Med 2015;372:793-5.

6. Jameson JL, Longo DL. Precision medicine-personalized, problematic, and promising. N Engl J Med 2015;372:2229-34.

7. Elliott P, Peakman TC. UK Biobank. The UK Biobank sample handling and storage protocol for the collection, processing and archiving of human blood and urine. Int J Epidemiol 2008;37:234-44.

8. Park MJ, Kim DH, Lim SH, et al. Features of gastric neoplasm detected during the screening examination. Gut Liver 2007;1:033-9.

9. Kim D, Chung GE, Kwak MS, et al. Body fat distribution and risk of incident and regressed nonalcoholic fatty liver disease. Clin Gastroenterol Hepatol 2016;14:132-8.

10. Chung SJ, Kim D, Song JH, et al. Comparison of detection and miss rates of narrow band imaging, flexible spectral imaging chromoendoscopy and white light at screening colonoscopy: a randomised controlled back-to-back study. Gut 2014;63:785-91.

11. Chung SJ, Park MJ, Kang SJ, et al. Effect of annual endoscopic screening on clinicopathologic characteristics and treatment modality of gastric cancer in a high-incidence region of Korea. Int $J$ Cancer 2012;131:2376-84.

12. Chung SJ, Kim YS, Yang SY, et al. Five-year risk for advanced colorectal neoplasia after initial colonoscopy according to the baseline risk stratification: a prospective study in 2452 asymptomatic Koreans. Gut 2011;60:1537-43.

13. Chung SJ, Lim SH, Choi J, et al. Helicobacter pylori serology inversely correlated with the risk and severity of reflux esophagitis in helicobacter pylori endemic area: a matched case-control study of 5616 health check-up Koreans. J Neurogastroenterol Motil 2011;17:267-73.

14. Chung SJ, Kim D, Park MJ, et al. Metabolic syndrome and visceral obesity as risk factors for reflux oesophagitis: a cross-sectional casecontrol study of 7078 Koreans undergoing health check-ups. Gut 2008;57:1360-5.

15. Chung GE, Kim D, Kim W, et al. Non-alcoholic fatty liver disease across the spectrum of hypothyroidism. J Hepatol 2012;57:150-6.

16. Chung GE, Choi SY, Kim D, et al. Nonalcoholic fatty liver disease as a risk factor of arterial stiffness measured by the cardioankle vascular index. Medicine 2015;94:e654.

17. Kim D, Choi SY, Park EH, et al. Nonalcoholic fatty liver disease is associated with coronary artery calcification. Hepatology 2012;56:605-13.

18. Kwak MS, Yim JY, Kim D, et al. Nonalcoholic fatty liver disease is associated with coronary artery calcium score in diabetes patients with higher HbA1c. Diabetol Metab Syndr 2015;7:28.

19. Choi SY, Kim D, Kim HJ, et al. The relation between non-alcoholic fatty liver disease and the risk of coronary heart disease in Koreans. Am J Gastroenterol 2009;104:1953-60.

20. Craig CL, Marshall AL, Sjöström M, et al. International physical activity questionnaire: 12 -country reliability and validity. Med Sci Sports Exerc 2003;35:1381-95.

21. Chun MY. Validity and reliability of Korean version of international physical activity questionnaire short form in the elderly. Korean J Fam Med 2012;33:144-51.

22. Kim WY, Cho MS, Lee HS. Development and validation of mini dietary assessment index for Koreans. Korean. J Nutr 2003;36:83-92

23. Beck AT, Steer RA, Carbin MG. Psychometric properties of the Beck Depression Inventory: twenty-five years of evaluation. Clin Psychol Rev 1988;8:77-100.

24. Wong ND, Hsu JC, Detrano RC, et al. Coronary artery calcium evaluation by electron beam computed tomography and its relation to new cardiovascular events. Am J Cardiol 2000;86:495-8.

25. Seidell JC, Bakker CJ, van der Kooy K. Imaging techniques for measuring adipose-tissue distribution--a comparison between computed tomography and 1.5-T magnetic resonance. Am J Clin Nutr 1990;51:953-7.

26. Bosch FX, Manos MM, Muñoz N, et al. Prevalence of human papillomavirus in cervical cancer: a worldwide perspective. International biological study on cervical cancer (IBSCC) Study Group. J Natl Cancer Inst 1995;87:796-802.

27. Kim MJ, Kim JJ, Kim S. Type-specific prevalence of high-risk human papillomavirus by cervical cytology and age: Data from the health check-ups of 7014 Korean women. Obstet Gynecol Sci 2013;56:110-20.

28. Yim JY, Kim N, Choi SH, et al. Seroprevalence of Helicobacter pylori in South Korea. Helicobacter 2007:12:333-40. 
29. Hamilton SR, Aaltonen LA. WHO classification of tumours of the digestive system. 3rd ed. Geneva: IARC, 2000.

30. Bosman FT, Carneiro F, Hruban RH, et al. WHO Classification of Tumours of the Digestive System. 4th ed. Geneva: IARC, 2010.

31. Eke PI, Dye BA, Wei L, et al. Update on prevalence of periodontitis in adults in the United States: NHANES 2009 to 2012. J Periodontol 2015;86:611-22.

32. White DA, Tsakos G, Pitts NB, et al. Adult dental health survey 2009: common oral health conditions and their impact on the population. Br Dent J 2012;213:567-72.

33. Murray H, Locker D, Kay EJ. Patterns of and reasons for tooth extractions in general dental practice in Ontario, Canada. Community Dent Oral Epidemiol 1996;24:196-200.

34. Lieberman DA, Rex DK, Winawer SJ, et al. Guidelines for colonoscopy surveillance after screening and polypectomy: a consensus update by the US Multi-Society Task Force on Colorectal Cancer. Gastroenterology 2012;143:844-57.

35. Chung SJ, Kim YS, Yang SY, et al. Prevalence and risk of colorectal adenoma in asymptomatic Koreans aged $40-49$ years undergoing screening colonoscopy. J Gastroenterol Hepatol 2010;25:519-25

36. Kang HY, Kim D, Kim HJ, et al. The relationship between colonoscopy procedure order and adenoma detection rates: a prospective study. J Clin Gastroenterol 2015;49:683-9.

37. Song JH, Kim SG, Chung SJ, et al. Risk of progression for incidental small subepithelial tumors in the upper gastrointestinal tract. Endoscopy 2015;47:675-9.

38. Chung SJ, Kim D, Song JH, et al. Efficacy of computed virtual chromoendoscopy on colorectal cancer screening: a prospective, randomized, back-to-back trial of fuji intelligent color enhancement versus conventional colonoscopy to compare adenoma miss rates. Gastrointest Endosc 2010;72:136-42.

39. Lim SH, Kwon JW, Kim N, et al. Prevalence and risk factors of Helicobacter pylori infection in Korea: nationwide multicenter study over 13 years. BMC Gastroenterol 2013;13:104.

40. Yang HJ, Lee C, Lim SH, et al. Clinical characteristics of primary gastric lymphoma detected during screening for gastric cancer in Korea. J Gastroenterol Hepatol 2016;31:1572-83.
41. Yang HJ, Lim SH, Lee C, et al. Management of suspicious mucosa-associated lymphoid tissue lymphoma in gastric biopsy specimens obtained during screening endoscopy. J Korean Med Sci 2016:31:1075-81.

42. Fan JG, Kim SU, Wong VW. New trends on obesity and NAFLD in Asia. J Hepatol 2017;67:862-73.

43. Bae JC, Cho YK, Lee WY, et al. Impact of nonalcoholic fatty liver disease on insulin resistance in relation to $\mathrm{HbA} 1 \mathrm{c}$ levels in nondiabetic subjects. Am J Gastroenterol 2010;105:2389-95.

44. Chang Y, Jung HS, Yun KE, et al. Cohort study of non-alcoholic fatty liver disease, NAFLD fibrosis score, and the risk of incident diabetes in a Korean population. Am J Gastroenterol 2013;108:1861-8.

45. Kim D, Kim WR. Nonobese fatty liver disease. Clin Gastroenterol Hepatol 2017; 15:474-85.

46. Alberti KG, Zimmet P, Shaw J, et al. The metabolic syndrome--a new worldwide definition. Lancet 2005;366:1059-62.

47. Nazare JA, Smith JD, Borel AL, et al. Ethnic influences on the relations between abdominal subcutaneous and visceral adiposity, liver fat, and cardiometabolic risk profile: the International Study of Prediction of Intra-Abdominal Adiposity and Its Relationship With Cardiometabolic Risk/Intra-Abdominal Adiposity. Am J Clin Nutr 2012;96:714-26.

48. Kwon HM, Kim BJ, Lee SH, et al. Metabolic syndrome as an independent risk factor of silent brain infarction in healthy people. Stroke 2006;37:466-70.

49. Kwak MS, Kim D, Chung GE, et al. Role of physical activity in nonalcoholic fatty liver disease in terms of visceral obesity and insulin resistance. Liver Int 2015;35:944-52.

50. Korea Ministry of Health and Welfare, Korea National Cancer Center. 2001 Annual Report of the Korea Central Cancer Registry: Korea National Cancer Center, 2003. https://www.e-crt.org/upload/pdf/crt36-19.pdf.

51. Whelton PK, Carey RM, Aronow WS, et al. ACC/AHA/AAPA/ ABC/ACPM/AGS/APhA/ASH/ASPC/NMA/PCNA guideline for the prevention, detection, evaluation, and management of high blood pressure in adults: a report of the American College of Cardiology/ American Heart Association Task Force on Clinical Practice Guidelines. J Am Coll Cardiol 2017;2017:41519-1. 